\title{
La crisis triguera en la provincia de Cádiz. Jerez de la Frontera, 1880-1900
}

\section{ENRIQUE MONTAÑES}

Desde comienzos de los años ochenta del siglo pasado, la agricultura gaditana sufrirá una agudización de sus problemas tradicionales por las dificultades experimentadas en sus dos cultivos fundamentales: el trigo y la vid. La importancia de estos cultivos la encontramos confirmada en las cifras que ofrecían los ingenieros agrónomos de la provincia (1).

\section{CUADRO I \\ LOS CULTIVOS DE LA PROVINCIA DE CADIZ}

\begin{tabular}{lrrrr} 
Cultivo & Has. & \% & Valor de la producción & \% \\
\hline Trigo & 100.500 & 54 & 16.833 .750 & 43 \\
Cebada & 28.000 & 15 & 3.570 .000 & 9 \\
Centeno & 170 & & 17.765 & \\
Maíz & 3.260 & 2 & 586.800 & 1 \\
Garbanzos & 15.500 & 8 & 2.712 .500 & 7 \\
Habas y otras leg. & 11.400 & 6 & 1.322 .400 & 3 \\
Total cere. y leg. & 158.830 & 85 & 25.043 .215 & 64 \\
Viñas & 20.646 & 11 & 6.968 .025 & 18 \\
Olivos & 12.646 & 7 & 1.643 .980 & 4 \\
Hortalizas y leg. & 3.475 & 2 & 5.560 .000 & 14 \\
$\quad$ TOTAL & 185.597 & & 39.215 .220 & \\
& & & &
\end{tabular}

(1) Datos del ingeniero Domingo Lizaur y Paúl en el documento titulado "Estado detallado de los gastos y productos de los cultivos que explota la Agricultura de esta provincia", de 1 de septiembre de 1888. Archivo del Ministerio de Agricultura, legajo 259. 
El papel determinante del trigo conllevaba la dependencia estrecha de la agricultura gaditana de las típicas oscilaciones en la producción en función de las oscilaciones meteorológicas. Pero a estas variaciones seculares se añadirían los problemas generados en la década de los ochenta del siglo pasado por la introducción de trigos extranjeros más baratos. Es la coyuntura que se conoce como la crisis triguera finisecular, que tendrá unas repercusiones directas en la agricultura gaditana. En este trabajo se pretende una primera aproximación al conocimiento del impacto de la crisis triguera en la provincia de Cádiz, rastreando sus síntomas y sus repercusiones tanto económicas como sociales.

En primer lugar hay que tener en cuenta que la producción triguera de la provincia estaba desigualmente repartida entre sus diferentes municipios, como nos confirman los datos del Catastro (2):

\section{CUADRO II}

\section{LA PRODUCCION TRIGUERA EN LA PROVINCIA DE CADIZ}

\section{Término municipal Has. cultivo Producción anual (H1.)}

\begin{tabular}{lrr}
\hline Alcalá Gazules & 5.429 & 41.846 \\
Alcalá Valle & 850 & 8.064 \\
Algar & 584 & 3.610 \\
Algeciras & 1.458 & 11.322 \\
Algodonales & 2.029 & 11.703 \\
Arcos & 10.242 & 81.022 \\
Los Barrios & 2.592 & 18.400 \\
Benaocaz & 683 & 3.767 \\
Bornos & 1.416 & 12.911 \\
El Bosque & 334 & 1.608 \\
Castellar & 930 & 5.877 \\
Conil & 549 & 4.600 \\
Espera & 3.089 & 28.208 \\
El Gastor & 533 & 4.761 \\
Grazalema & 687 & 3.339 \\
Chiclana & 1.021 & 8.478 \\
Jerez & 20.930 & 197.752 \\
& & \\
(2) Memoria Resumen del Catastro Agronómico de Cádiz. Archivo Histórico Provincial \\
$\quad$ de Cádiz, Sección de Hacienda, legajo 507.
\end{tabular}


Término municipal

Jimena

Medina

Olvera

Paterna

El Pto. Sta. M.

Prado del Rey

Puerto Real

Puerto Serrano

Rota

Sanlúcar

San Roque

Setenil

Tarifa

Torre Alháquime

Trebujena

Ubrique

Vejer

Villaluenga

Villamartín

Zahara'
Has. cultivo

Producción anual (HI.)

4.624

7.901

4.215

416

1.753

1.140

733

204

839

1.017

2.131

1.698

8.395

415

754

833

4.686

420

4.590

897
25.861

63.474

48.115

2.296

18.248

9.936

7.750

1.108

7.391

11.507

11.148

17.035

52.260

5.203

8.530

4.235

35.470

2.127

24.952

8.055

Esta y otras estadísticas nos confirman a Jerez como el gran centro agrícola de la provincia que en cierto sentido marcaba la pauta al resto de las poblaciones. Este hecho permite elegir el estudio de la agricultura jerezana, apoyado además en una mayor disponibilidad y abundancia de fuentes, como un buen indicador que nos muestra en líneas generales la evolución global de toda la provincia.

\section{LA AGRICULTURA JEREZANA}

En primer lugar no resulta nada difícil establecer con exactitud la distribución de la tierra cultivada. Hemos de atenernos a los diferentes datos, normalmente de origen fiscal, para establecer una cierta aproximación que podríamos sintetizar en este cuadro (3):

(3) Los datos de 1876 son del expediente sobre la Estadística para el impuesto de Consumos. Archivo Municipal de Jerez de la Frontera, legajo 269, expediente 8.200. Los datos de 1898 corresponden a los amillaramientos, y los de 1899 al nuevo Catastro, y se pueden encontrar en el Boletín de la Cámara Agraria de Jerez de la Frontera, n.o 82, 31 de julio de 1899 , p. 160. 


\section{CUADRO III \\ LA DISTRIBUCION DE LA TIERRA CULTIVADA EN JEREZ}

\begin{tabular}{lrrr} 
Cultivo & $\mathbf{1 8 7 6}$ & $\mathbf{1 8 9 8}$ & $\mathbf{1 8 9 9}$ \\
\hline Cereal & 65.000 & 64.752 & 72.140 \\
Viñas & 5.950 & 6.178 & 7.760 \\
Olivar & 1.257 & 1.253 & 2.361 \\
Dehesas, monte, pastos & & 54.658 & 50.959 \\
Regadí & & 93 & 289
\end{tabular}

Estas y otras cifras similares hay que tomarlas como puntos de referencia más que como datos exactos. Quizás sean las proporcionadas por el Catastro de 1899 las que más se correspondan con la realidad, ya que fue el esfuerzo institucional más serio realizado para medir la riqueza rústica del país. De hecho afloraron más de 10.000 hectáreas de tierra cultivada que la anterior documentación fiscal, los amillaramientos, no recogía, lo que supuso un 10\% de aumento.

Respecto al valor total de las principales producciones agrícolas podemos realizar algunas extrapolaciones a partir de datos aislados y fragmentarios que nos aproximan al problema. Tomando los cálculos elaborados por el ingeniero agrónomo provincial Domingo Lizaur y Paúl para 1888 sobre el valor de la producción agrícola para toda la provincia y la participación de Jerez en ella según los diferentes cultivos, podemos establecer las siguientes cifras (4):

\section{CUADRO IV \\ VALOR DE LAS PRODUCCIONES AGRICOLAS DE JEREZ}

\section{Cultivo}

Trigo

Cebada

Centeno
$\%$ sobre el total prov.

37

14

18
Valor producción

5.566 .000

499.800

3.197

(4) La participación de los cultivos de Jerez en el total provincial está calculada a partir de los datos que se encuentran en: Dirección General de Agricultura, Industria y Comercio. Avance estadístico sobre el cultivo del cereal y de leguminosas asociados en España formado por la Junta Consultiva Agronómica. Quinquenio 18861890. Madrid, 1891. Para el valor de la producción he tomado como referencia los cálculos antes citados del ingeniero agrónomo provincial Domingo Lizaur. 


$\begin{array}{lrr}\text { Garbanzos } & 30 & 813.750 \\ \text { Habas } & 37 & 489.288 \\ \text { Vid } & 30 & 2.090 .407 \\ \text { Olivo } & 10 & 164.398\end{array}$

Aun cuando todos esos componentes no eran los únicos elementos del ingreso agrario, faltan otros importantes capítulos como los aprovechamientos ganaderos y forestales; los datos del cuadro nos confirman la decisiva importancia del trigo y la viña como pilares sobre los que se asentaba la buena marcha de la agricultura jerezana y también provincial. Precisamente los problemas que sufrieron ambos cultivos a partir de la década de los ochenta influirán en el declive de todo el sector.

\section{EL IMPACTO DE LA CRISIS TRIGUERA}

El cultivo del cereal se vio inmerso en la comúnmente denominada crisis finisecular (5). Se sufrió en toda Europa a partir de la década de los setenta, y su manifestación más visible fue el descenso del precio del trigo. Esta caída fue originada por la irrupción de cereales originarios de América y otros lugares, que gracias a una reducción de los precios del transporte pudieron situar en Europa producciones más baratas.

Era un proceso impulsado por el mismo desarrollo del capitalismo, que iba consolidando los fundamentos de un mercado mundial. Esta internacionalización de la economía provocaba que las produccio-

(5) Sobre la crisis agraria de fin de siglo destaquemos los trabajos de: GARRABOU, R. (ed): La crisis agraria de fines del siglo XIX. Crítica, Barcelona, 1988. BERNAL, A.M.: La llamada crisis finisecular, 1872-1919. En GARCIA DELGADO, J.L. (ed.): La España de la Restauración. Política, economía, legislación y cultura. Siglo XXI, Madrid, 1985. GARRABOU, R.: La crisis agraria española de finales del siglo XIX: una etapa del desarrollo del capitalismo. En GARRABOU, R. y SANZ, J. (eds.): Historia agraria de la España contemporánea. Crítica, Barcelona, 1985, vol. 2, pp. '477-542. SANZ FERNANDEZ, J.: La crisis triguera finisecular: los ultimos años. En GARCIA DELGADO, J.L. (ed.): La España de la Restauración. Política, economia, legislación y cultura. Siglo XXI, Madrid, 1985, pp. 266-310. BERNAL, A.M.: Resistencias al cambio económico desde el sector agrícola (1880-1931). En GARCIA DELGADO, J.L. (ed.): España entre dos siglos (1875-1931). Siglo XXI, Madrid, 1991, pp. 140-156. 
nes más baratas de países alejados, obtenidas gracias a una mayor eficiencia o por contar con menores costes merced a un menor precio de la tierra o a una sobrexplotación campesina, pusieron en crisis las explotaciones tradicionales de algunos países europeos que habían atravesado durante gran parte del siglo XIX una cierta etapa expansiva.

La caída del precio de los cereales desvalorizaba considerablemente la producción obtenida, y situaba a buen número de explotaciones en condiciones precarias para subsistir en una economía de mercado. Como ha señalado R. Garrabou, la crisis empezó a afectar a España con cierto retraso respecto a Europa a partir de la década de los ochenta, y lo hizo en principio de forma moderada.

Esta tendencia general tuvo en Jerez sus manifestaciones, que es necesario esclarecer, no solamente a partir de la estadística de los precios, sino también teniendo en cuenta la de las producciones, para obtener una imagen más global de las repercusiones de la crisis (6).

\section{CUADRO $\mathrm{V}$ \\ LOS PRECIOS DEL TRIGO}

\begin{tabular}{lcccc} 
Años & Jerez & Indice & España & Indice \\
\hline $1875-1879$ & 24.6 & 100 & 24.3 & 100 \\
$1880-1884$ & 24.9 & 101 & 24.2 & 99 \\
$1885-1889$ & 19.9 & 81 & 21.2 & 87 \\
$1890-1894$ & 21.9 & 89 & 21.5 & 88
\end{tabular}

Como puede apreciarse, en el primer quinquenio de los ochenta los precios no descendieron, tanto porque hubo dos años de escasísima cosecha (1881 y 1882) como por la existencia de un fuerte arancel para los trigos extranjeros (6,98 ptas/Hl.) desde 1887. Sin embargo, entre 1885 y 1889 los precios descendieron un $20 \%$, no solamente porque la cosecha fue superior en un $18 \%$, sino también porque aumentaron apreciablemente las entradas de granos extranjeros.

Precisamente en estos años se redoblaron las protestas y la agitación de los labradores y propietarios agrícolas tanto en Castilla como en Andalucía, en demanda de una mayor protección por parte de los poderes públicos. El gobierno organizó una gran encuesta nacional, se

(6) Los precios de Jerez son del Boletín de la Cámara Agraria, n. 27, julio 1896, p. 276. Los de España, GEHR.: Sector agrario. En Estadísticas Históricas de España, siglos $X I X-X X$. Fundación Banco Exterior, Madrid, 1989, p. 115. 
publicaron informes, se reunieron comisiones, etc. (7). Todo lo cual preparó el terreno para que el gobierno conservador aumentara el arancel triguero en 1891.

En el quinquenio 1890-94, el reforzamiento del arancel tuvo sus efectos y los precios subieron un 10\%, aunque esta tendencia también se sustentó en las malas cosechas de 1891, 1892 y 1893, que hicieron descender la producción media del quinquenio en un $14 \%$. La gran sorpresa y disgusto de los agricultores era muy ostensible, porque a pesar de todo ello el precio no subió todo lo que se podía esperar de una serie de años tan malos, ni tan siquiera recuperó el nivel del quinquenio 1880-84.

Esta situación marcaba una diferencia clara con respecto a crisis anteriores, por cuanto parecían desaparecer las alzas cíclicas de los años de escasez. La permanente entrada de trigos extranjeros provocada por la consolidación de un mercado mundial atemperaron las subidas de los precios en años de tan escasa cosecha como el de 1892. Esta nueva circunstancia limitó la efectividad de una práctica secular como la del almacenamiento de grano en espera de un período de precios altos. La Cámara Agraria de Jerez reconocía que a pesar de ser Cádiz una provincia deficitaria existían cantidades importantes de grano almacenado, por imperar en el mercado precios considerados como ruinosos a consecuencia de la entrada de los cereales extranjeros:

Algunas estadísticas nos permiten registrar esa tendencia creciente de las importaciones a pesar incluso del arancel de 1891 (8).

\section{CUADRO VI \\ IMPORTACIONES DE TRIGO POR MAR EN LA PROVINCIA DE CADIZ (HI.)}

\begin{tabular}{llll} 
Años & Trigo & Harina & Total \\
\hline $1880-86$ & 50.050 & 24.536 & 74.586 \\
$1890-94$ & 69.000 & 16.256 & 85.256
\end{tabular}

(7) El material recopilado en la encuesta nacional está recogido en varios volúmenes con el título de La crisis agraria y pecuaria, Madrid, 1887-1888-1889.

(8) Los datos se pueden encontrar en el Informe acerca de la producción y comercio del trigo y su harina en la provincia de Cádiz, en el Boletín de la Cámara Agraria de Jerez, n. ${ }^{\circ} 17$, septiembre de 1895 . También en el n. ${ }^{\circ}$ 31, noviembre de 1896, p. 331, del mismo Boletín. 
Las referidas cantidades de trigo extranjero significaban una parte importante de las importaciones netas totales de toda la provincia: un $34 \%$ en el período $1880-86$ y un $30 \%$ en el de $1890-94$. Lo cual nos muestra la parcela del mercado que el trigo extranjero había conseguido conquistar pese a los obstáculos proteccionistas.

La caída de los precios significaba la disminución de los ingresos para los agricultores. Para apreciar mejor la entidad de esta pérdida hay que combinar la evolución de los precios y la de la producción (9).

\section{CUADRO VII \\ VALOR DE LA PRODUCCION TRIGUERA DE JEREZ}

\begin{tabular}{lccrc} 
Años & Producción & Indice & Valor & Indice \\
\hline 1879 & 375.000 & 164 & 10.275 .000 & 181 \\
$1880-1884$ & 227.400 & 100 & 5.662 .260 & 100 \\
$1885-1889$ & 270.000 & 119 & 5.373 .000 & 95 \\
$1890-1894$ & 232.000 & 102 & 5.080 .800 & 88
\end{tabular}

Desde 1880 se asiste a una progresiva desvalorización de la producción triguera que resulta casi imparable incluso con la presencia del nuevo arancel de 1891. Esta tendencia sumía en grandes dificultades a los agricultores de todos los tamaños y comprometía seriamente la permanencia de sus explotaciones dentro de las pautas que marcaba la evolución del mercado.

Esta inadaptación a las nuevas condiciones impuestas por la internacionalización del mercado triguero ponía en evidencia la falta de eficiencia de la producción cerealista, y su anquilosamiento en los métodos de cultivo tradicionales. Realidad que provocó un amplio debate social en el que se trató de poner en evidencia las causas que producían el retraso de la agricultura española. En este sentido, la mirada de los contemporáneos se dirigió hacia todos aquellos factores que incidian en las condiciones de producción.

Así, propietarios y labradores hacían recaer la máxima responsabilidad de la situación sobre el excesivo volumen de impuestos que soportaba la actividad agraria, o bien en los costes laborales considerados como muy altos.

(9) Las cosechas entre 1879 y 1894 en Boletín de la Cámara Agraria, n. ${ }^{\circ} 6$, octubre de 1894, p. 56. Las cosechas entre 1862-1866 en el Archivo Municipal de Jerez, legajo 268, expediente 8.193. Los precios en el Boletín de la Cámara Agraria, n.․ 27, julio 1896, p. 276 
Por el contrario, políticos e intelectuales de corte reformista insistían en otros problemas estructurales, como la bajísima productividad del trabajo por el empleo de métodos anticuados, o en el desigual reparto de la tierra, que generaba un peso excesivo de los grandes propietarios que fundamentalmente ejercían de rentistas. La significación de cada una de estas variables dentro del contexto general de la crisis sigue constituyendo un debate abierto entre los historiadores, que necesariamente han de ampliar el conjunto de evidencias para alcanzar conclusiones más sólidas.

\section{LA PERSISTENCIA DE LAS TECNICAS TRADICIONALES}

El primer aspecto a considerar tiene que ser el de las formas y métodos de cultivo. Los testimonios localizados sobre la década de los sesenta y la de los setenta del siglo pasado coinciden en señalar la presencia dominante del cultivo del cereal al tercio (10). De acuerdo con esta práctica tradicional se dividía la superficie en tres hojas o partes: una de ellas se reservaba para el cultivo del cereal (sobre todo trigo, y en menor medida cebada), otra se dejaba en barbecho y la última se convertía en zona de dehesa para pastos o manchón.

Este era un sistema de rotación trienal general sobre el que podían realizarse muchas variaciones en la práctica. Por ejemplo, en el cultivo al tercio extensivo era en el que se cultivaba estrictamente un tercio de la superficie. Sin embargo, el tercio intensivo era una forma más evolucionada que podía llegar a cultivar casi por completo la hoja de barbecho con leguminosas o plantas forrajeras, por lo tanto ocupaba las dos terceras partes de la superficie. Era una forma de transición hacia el cultivo anual, en el que se eliminaba definitivamente el barbecho distribuyendo la superficie en dos hojas: una para el cereal y otra para plantas barbecheras (leguminosas, remolacha, etc.) (11).

El reto para una agricultura cerealista poco eficiente era el de obtener mejores resultados tratando de intensificar el cultivo. Por lo tanto, uno de los indicadores que se vienen considerando para medir la modernización de la agricultura es el del paso del cultivo al tercio al anual. Resulta difícil medir con los datos que poseemos el momento de ese cambio.

(10) Archivo Municipal de Jerez, Protocolos n.․ 163, 1872.

(11) Sobre el largo período de transición desde el cultivo al tercio hasta el de año y vez se puede consultar: SUMPSI, J.M.: Estudio de la transformación del cultivo al tercio al de año y vez en la campiña de Andalucía. Agricultura y Sociedad, n. 6, 1978. 
Los informes de los ingenieros agrónomos de la provincia de Cádiz y de Jerez mismo coincidian en la presencia mayoritaria del tercio extensivo a comienzos de los ochenta (12). Además, entendían que era la única forma de cultivo posible debido a la poca envergadura del capital de explotación de la mayor parte de los agricultores.

La utilización generalizada de los llamados "motores de sangre" (hombres y animales) con unos aperos de labranza demasiado simples que daban como resultado unas labores poco profundas, junto con el escaso empleo de los abonos, incluso de los de origen animal, determinaban la imposibilidad de abandonar el cultivo al tercio. Las limitaciones de esta forma de cultivo propiciaban una menor productividad por hectárea, que sólo mejoraba en función de las condiciones meteorológicas en los años de buenas cosechas.

Si en lo que respecta a la división de la superficie cultivable y a la preparación de la tierra con sus labores de arado y siembra dominaban los métodos tradicionales, sin embargo también diversos observadores resaltaron que en otras facetas sí que se introdujeron novedades como las máquinas segadoras o trilladoras desde fechas tan tempranas como la década de los sesenta.

En 1863, una sociedad integrada por medio centenar de agricultores jerezanos, el "Fomento Agrícola", impulsó y promocionó el uso de ese tipo de mecanización demostrando sus ventajas en algunas experiencias concretas. La iniciativa fue apoyada por grandes propietarios como los Guerrero, y resulta difícil valorar su alcance y difusión en todo el término jerezano por carecer de datos concretos.

En cualquier caso, parece claro que la introducción de este tipo de maquinaria está estrechamente ligada con la existencia de grandes explotaciones que gracias a su economía de escala podían rentabilizar la inversión. Recordemos que en el término municipal de Jerez de la Frontera las fincas mayores de 200 has. representaban más del setenta por ciento de su extensión (13).

\section{LAS RESPUESTAS A LA CRISIS}

Un tema central entre todos los historiadores de la crisis agraria de fin de siglo es precisamente el de los cambios que se pudieron

(12) Memoria sobre el estado de la Agricultura en la provincia de Cádiz y medios de mejorarla, 1875, Archivo del Ministerio de Agricultura, legajo 25.

(13) Sobre los comienzos de la mecanización en Jerez: FERNANDEZ DE LA ROSA, G. y otros: Fomento agricola en Jerez de la Frontera. Jerez, 1864. 
generar desde la década de los años ochenta, como consecuencia de los problemas abiertos por el descenso del precio del cereal. Si esos cambios se orientaron hacia la modernización de la agricultura en el marco de una economía de mercado cada vez más internacionalizada es el punto de partida de la mayor parte de los análisis.

En este sentido, es necesario plantearse si las características de la explotación cerealista de Jerez a comienzos de los ochenta sufrió alguna transformación significativa hasta fin de siglo.

Los informes y datos acumulados para la elaboración del Catastro en 1899 quizás constituyan la mejor fuente para reconstruir una fotografía del estado de la agricultura gaditana. En el informe del director provincial de los trabajos catastrales, de nuevo se resalta el absoluto predominio del sistema trienal ya que era “... muy racional y estaba en armonía con el escaso capital de explotación que interviene" (14).

Otros testimonios relacionados directamente con Jerez también insistían en la presencia dominante del sistema al tercio. De hecho, un importante propietario como el marqués de Bertemati reconocía que se hacía urgente "...la necesidad de transformar radicalmente los rutinarios y expoliadores sistemas de tres hojas, de barbechos y erías seguidos hasta aquí, para que nuestra agricultura abandone los moldes de los tiempos de los moriscos y entre en el concierto de los pueblos civilizados" (15).

Parece, por lo tanto, que la crisis no impulsó la intensificación de los cultivos y que la evolución desde el sistema trienal al anual parecía lejos de haberse iniciado con firmeza. Tanto las hojas declaratorias del Catastro como los datos que conocemos sobre producciones y superficies cultivadas ratifican esa observación. Es más, parece que la superficie anual cultivada de trigo, que según los cálculos de la Cámara Agrícola de Jerez se cifraba en 22.000 has. para los años ochenta y principios de los noventa, se redujo a 20.000 has: de acuerdo con los datos proporcionados por el director provincial de los trabajos catastrales en su informe citado.

El descenso del precio del trigo provocó el abandono de las peores tierras y agudizó otro de los defectos endémicos de la agricultura gaditana: la escasez del capital de explotación.

En estas circunstancias no fueron muy numerosas las iniciativas tendentes a modernizar la actividad buscando una mejora de la pro-

(14) Memoria Resumen del Catastro Agronómico de Cádiz, Archivo Provincial de Cádiz, Sección de Hacienda, legajo 507.

(15) El Guadalete, 23 agosto 1902. 
ductividad. En lo que respecta a las labores de preparación de la tierra apenas tenemos noticias de modificaciones. Unicamente en el Boletín de la Cámara Agrícola aparece una intensa propaganda en favor de los abonos minerales, que pregona sus grandes ventajas a partir de pequeñas experiencias que se van realizando en la zona.

Pero seguían siendo casos aislados y de poca incidencia en el conjunto general de la actividad agrícola. Por ello no es extraño que la productividad por ha. permaneciera prácticamente estancada y que tan sólo ạumentara gracias a las buenas cosechas de los años con una meteorología favorable (16).

\section{CUADRO VIII RENDIMIENTOS DEL TRIGO EN JEREZ (H1/Ha.)}

\begin{tabular}{lcc} 
Años & Hi/Ha. & Indice \\
\hline $1862-1866$ & 9.8 & 100 \\
$1880-1884$ & 10.3 & 105 \\
$1885-1889$ & 12.2 & 124 \\
$1890-1894$ & 10.5 & 107
\end{tabular}

En cuanto a la continuidad en el esfuerzo por mecanizar labores como la siega y la trilla, que tan prematuramente se había iniciado en los años sesenta, no poseemos unos datos muy precisos para valorarla hacia fines de siglo. Al parecer, las máquinas segadoras actuaban en alrededor de la cuarta parte de la superficie cultivada de cereales.

Según escribía el director de la Granja Experimental de Jerez, Gumersindo Fernández de la Rosa, "...no cabe decir que el empleo de las segadoras mecánicas sea dominante en la localidad; y sin embargo, pocos serán los pueblos agrícolas de alguna importancia en que se llegue a la indicada proporción" (17).

Sobre las trilladoras aún tenemos menos referencias completas. Cabe deducir, por las bases de trabajo vigentes durante las labores de trilla, que la faena con máquinas estaba extendida puesto que, por ejemplo, los salarios para los que trabajaban con ellas figuraban siempre en todas las ofertas de los labradores. Quizás este sea un indica-

(16) Se ha tomado como superficie media cultivada durante todo el período la de 22.000 has. El volumen de las cosechas de cada año en el Boletín de la Cámara Agraria, $n . \circ 6$, octubre 1894 , p. 56 .

(17) Boletín de la Cámara Agraria de Jerez, n. 16, noviembre 1895, p. 167. 
dor que nos muestra el arraigo de las trilladoras y que coincidiría con la apreciación de Simpson de que en Andalucía las trilladoras tendrían una mayor acogida que las segadoras (18).

Cuando dispongamos de más datos concretos podremos calibrar mejor la incidencia de esta incipiente mecanización. En cualquier caso, la desvalorización de la producción agrícola que trae la crisis no parece que hiciera posible grandes inversiones en maquinaria. Sólo grandes propietarios como los Guerrero tenían un conjunto de máquinas significativo.

En términos generales parece, pues, que el escaso aumento del capital de explotación no pudo generar una intensificación del cultivo ni una modernización de la actividad. Las respuestas se encaminaron a disminuir los costes 'de producción por diferentes vías: abandono de tierras marginales, reducción de labores, congelación salarial, etc. Actitudes conservadoras todas ellas, que no aumentaban apreciablemente la productividad y que generaban unas repercusiones sociales apreciables al disminuir la ocupación agrícola.

El capítulo de los salarios fue uno de los que principalmente se trató de reducir o congelar. De hecho, entre 1883 y 1902 no parece que los salarios sufrieran ninguna variación e incluso tendieron a bajar en algunos casos ante la caída de la actividad y la abundante oferta de mano de obra.

Como prueba de esta congelación salarial baste citar que las labores de trilla a máquina en 1883 se retribuían con cuatro reales y que en 1902 el Gremio de Labradores seguía ofertando la misma cantidad.

Este descenso o estancamiento de los salarios se vio facilitado por la escasa disminución de la población activa agraria, que posibilitaba una abundante oferta de mano de obra ante un número más reducido de labores agrícolas (19).

(18) SIMPSON, J.: La elección de técnica en el cultivo triguero y el atraso de la agricultura española a finales del siglo XX. Revista de Historia Económica, Año V, n. ${ }^{2}$, 1987, pp. 271-299.

(19) Censos de Población de 1887 y 1900. 


\section{CUADRO IX \\ LA EVOLUCION DE LA POBI.ACION ACTIVA AGRARIA EN LA PROVINCIA DE CADIZ, 1887-1900}

\begin{tabular}{lrrrr} 
Población & $\mathbf{1 8 8 7}$ & $\mathbf{\%}$ & $\mathbf{1 9 0 0}$ & $\mathbf{\%}$ \\
\hline Varones & 84.158 & 97 & 83.005 & 97 \\
Mujeres & 2.412 & 3 & 2.815 & 3 \\
Total & 86.570 & 52 & 83.005 & 52 \\
Menores de 20 años & 16.217 & 19 & 13.620 & 16
\end{tabular}

Entre estos trece años en que disponemos de cifras comparativas la población activa agraria disminuyó en un cuatro por ciento, pero hay que tener en cuenta que el conjunto de la población activa descendió aún más, un 5\%, por lo que el porcentaje de la población activa agraria se mantuvo sin variación, un $52 \%$, superando a la suma del resto de los sectores productivos. El grupo de edad que soportó el mayor descenso en la ocupación agraria, un 73\%, fue el de menores de 20 años, que refleja las dificultades de los más jóvenes para ocuparse en una actividad en crisis.

Al lado de esta reducción en los gastos salariales, también los propietarios y agricultores presionaron para reforzar las barreras proteccionistas. Su influencia política en el Estado de la Restauración obtuvo una subida casi permanente del arancel para defender la rentabilidad de sus explotaciones (20).

\section{CUADRO X \\ EVOLUCION DE LOS ARANCELES SOBRE EL TRIGO, 1880-1900}

\begin{tabular}{cc} 
Años & \multicolumn{2}{c}{ Arancel (Ptas/Qm.) } \\
\hline 1882 & 5.70 \\
1891 & 8 \\
1899 & 8
\end{tabular}

Para la Cámara Agraria de Jerez, el logro de un precio remunerador para el trigo, que cifraba alrededor de las 22 ptas/Hl., debía obte-

(20) Ver a este respecto el trabajo de SERRANO SANZ, J.M.: El viraje proteccionista en la Restauración. La política comercial española, 1875-1895. Siblo XXI, Madrid, 1987. La tabla de los aranceles pertenece a esta obra, p. 98. 
nerse con un control más riguroso de las aduanas y por la aplicación sistemática del arancel (21).

La defensa del arancel, no sólo era fundamental para los grandes propietarios y agricultores, sino sobre todo era imprescindible para los pequeños propietarios, y campesinos, que en principio eran los más afectados por la desvalorización de la producción agrícola. Parece lógico pensar que parte del campesinado aceptó la tutela política de los grandes propietarios, como forma de defender sus pequeñas explotaciones de la penetración de una economía de mercado que conllevaba su desaparición.

Sin embargo, otros grupos de campesinos y jornaleros protagonizaron en un par de ocasiones (la huelga de jornaleros en 1883 y el asalto de Jerez en 1892) una lucha social radicalizada de evidentes resonancias políticas que, aunque no tuvo frutos a corto plazo y las organizaciones de jornaleros acabaron disueltas, abonará las expectativas del reformismo agrario, que presentará sus alternativas como única forma para resolver la crisis agraria.

(21) Boletín de la Cámara Agraria de Jerez, n.ำ 17, septiembre 1895. 